\title{
Microvascular decompression for trigeminal neuralgia: comments on a series of 250 cases, including 10 patients with multiple sclerosis
}

\author{
Giovanni Broggi, Paolo Ferroli, Angelo Franzini, Domenico Servello, Ivano Dones
}

\begin{abstract}
Objective-To examine surgical findings and results of microvascular decompression (MVD) for trigeminal neuralgia (TN), including patients with multiple sclerosis, to bring new insight about the role of microvascular compression in the pathogenesis of the disorder and the role of MVD in its treatment.

Methods-Between 1990 and 1998, 250 patients affected by trigeminal neuralgia underwent MVD in the Department of Neurosurgery of the "Istituto Nazionale Neurologico C Besta" in Milan. Limiting the review to the period 1991-6, to exclude the "learning period" (the first 50 cases) and patients with less than 1 year follow up, surgical findings and results were critically analysed in 148 consecutive cases, including 10 patients with multiple sclerosis.
\end{abstract}

Results-Vascular compression of the trigeminal nerve was found in all cases. The recurrence rate was $15.3 \%$ (follow up 1-7 years, mean 38 months). In five of 10 patients with multiple sclerosis an excellent result was achieved (follow up 12-39 months, mean 24 months). Patients with TN for more than 84 months did significantly worse than those with a shorter history $(p<0.05)$. There was no mortality and most complications occurred in the learning period. Surgical complications were not related to age of the patients.

Conclusions-Aetiopathogenesis of trigeminal neuralgia remains a mystery. These findings suggest a common neuromodulatory role of microvascular compression in both patients with or without multiple sclerosis rather than a direct causal role. MVD was found to be a safe and effective procedure to relieve typical TN in patients of all ages. It should be proposed as first choice surgery to all patients affected by $T N$, even in selected cases with multiple sclerosis, to give them the opportunity of pain relief without sensory deficits.

(F Neurol Neurosurg Psychiatry 2000;68:59-64)

Keywords: trigeminal neuralgia; microvascular decompression; multiple sclerosis

Dr Giovanni Broggi,

Department of

Neurosurgery, Istituto

Nazionale Neurologico C

Besta, Via Celoria 11, 20133

Milan, Italy

Received 17 June 1998 and in final form

30 July 1999

Accepted 11 August 1999
The concept of microvascular compression of the trigeminal nerve described by Dandy in $1934,{ }^{1}$ rediscovered by Gardner and Miklos, and fully recognised and popularised by Jannetta ${ }^{3}$ was a milestone in the management of medically intractable trigeminal neuralgia. In the past 30 years thousands of patients have undergone successful microvascular decompression and today it represents one of the most widely used surgical options for trigeminal neuralgia. Several studies agree on high rate of long term success and even authors against the concept of microvascular compression perform it for its effectiveness. ${ }^{4}$ Controversies still exist about the role of vascular compression in the pathogenesis of the disorder, the possible involvement of the same mechanism also in patients affected by multiple sclerosis, the existence of reliable prognostic factors, and the role of microvascular decompression in elderly patients.

To bring new insight about these topics we critically reviewed 250 patients, including 10 patients affected by multiple sclerosis, all operated on by the same surgeon (GB).

\section{Patients and methods}

Between 1990 and 1998, 250 patients affected by trigeminal neuralgia (TN) underwent microvascular decompression (MVD) in the Department of Neurosurgery of the "Istituto Nazionale Neurologico C. Besta" in Milan. Our review was limited to 146 patients treated in the period 1991-October 1996. Those patients with less than 1 year follow up and the first 50 cases (the "learning period"), in which a slightly different surgical technique was used, were excluded. In 10 cases TN was associated with multiple sclerosis, clinically diagnosed and proved by MRI and CSF examination.

Individual symptoms, clinical history, operative findings, and complications were recorded. Operative results were assessed by clinical follow up and periodic phone surveys. The outcome of intervention was graded by an independent reviewer other than the operating surgeon.

All of these patients had been given previous medical therapy (carbamazepine, diphenilhydantoine, baclofen), to which they had become refractory or intolerant. Contrast enhanced CT and MRI were routinely performed to exclude cerebellopontine angle mass lesions, to find signs of demyelinating disease or neurovascular compression. As most cases had had MRI in other hospitals, sequences varied and specific studies such as magnetic resonance tomographic angiography were used in only 17 patients. The presence of a demyelinating plaque in the trigeminal root entry zone (REZ), pontine tract, and nuclei was considered a contraindication to open surgery. The 
failure to image microvascular compression with MRI was not considered sufficient to exclude patients from MVD, because of the possibility of false negatives.

SURGICAL TECHNIQUE

Exploration of the cerebellopontine angle was performed by GB through a small, keyhole type (less than $20 \mathrm{~mm}$ diameter) retromastoid craniectomy, with patients in the supine position with the head rotated to the side opposite the neuralgia. The margins of transverse sinus and sigmoid sinuses were exposed and the dura was opened along the line bisecting their angle. The fifth cranial nerve was exposed through a supracerebellar approach, thus avoiding lateral retraction of the cerebellar hemisphere and traction of the VII-VIII complex of cranial nerves. Neither lumbar CSF drainage nor mannitol were used to avoid anatomical modification before dural opening. In approaching the trigeminal nerve, care was taken to spare at least two petrous veins. The nerve was cautiously dissected free without unnecessary manipulation. Any compressive arteries were relocated away from the trigeminal nerve and its root entry zone in the pons by the use of small pieces of teflon. Compressive veins were electrocoagulated and divided. In the "learning period" a greater craniectomy (about $35 \mathrm{~mm}$ diameter), a wider dural opening, and a lateral cerebellar approach with a self retaining retractor were used. As previously reported, ${ }^{5}$ brain stem auditory evoked potentials were recorded in 98 cases (37 during the "learning period" and 61 in subsequent cases).

STATISTICAL ANALYSIS

A statistical analysis of "essential" TN group $(n=136)$ was used to relate likelihood of postoperative recurrence of tic to the following variables: patient's age and sex; involved side and branch; duration of symptoms; history of previous trigeminal ablative procedures; type of neurovascular compression (arterial, venous, or both); postoperative numbness, and hypertension. Continuous data were compared using a two sample $t$ test; discrete data were compared using $\chi^{2}$ or Fisher's exact test, as appropriate. Recurrence free curves were compared by log rank test.

\section{Results}

The follow up ranged from 1 to 7 years (mean 38 months). The report includes 75 female patients and 71 male patients ranging in age from 20 to 76 years (mean 56 years). The right side of the face was affected in 81 cases and the left side in 67. In two patients both sides of the face were involved at different times. The branch distribution to which pain was referred was: V1 only, one case $(0.6 \%)$; V2 only, 26 cases $(17 \%)$; V3 only, 32 cases $(21,4 \%)$; V1 and V2, 30 cases $(20.5 \%)$; V2 and V3, 43 cases $(29,5 \%)$; and V1, V2, and V3, 16 cases $(11 \%)$. Duration of preoperative symptoms ranged between 3 months and 35 years (mean 8.5 years). Half of the patients had TN for more than 7 years. Sixty six patients had undergone previous procedures affecting the nerve such as radiofrequency thermal rhizotomy, balloon microcompression, glycerol rhizotomy, alchool injection, or peripheral avulsion (one procedure in 35 , two in 16 , three in five, four in two, and five in two patients). The longer the clinical history, the more likely that previous procedures had been performed and more branches were involved. Arterial hypertension was associated with trigeminal neuralgia in 40 patients $(27 \%)$. Two patients had bilateral $\mathrm{TN}$, so 148 affected sides were initially operated on. Seven patients required a second operation for a total of 155 MVDs. At the first operation a microvascular compression at the trigeminal REZ was found in all patients: severe, with a clear groove on the trigeminal root in 133 cases, slight, with a vascular contact not distorting the root in 15 . In 138 cases of "essential" TN it was arterial in 70 , venous in 18 , and both arterial and venous in 50. In 10 patients with multiple sclerosis it was arterial in five, venous in three, and arteriovenous in two. In 12 patients (three with multiple sclerosis) an arachnoiditis coexisted; in four cases vascular compression occurred well beyond the REZ.; in 13 the trigeminal nerve seemed to be shorter than usual and in five an asymptomatic microvascular compression of the VII-VIII complex was found.

The stabbing paroxysmal pain typical of trigeminal neuralgia disappeared immediately after surgery in $126(85 \%)$ out of 148 initially operated sides and faded away within 2 weeks in another $11(7.5 \%)$. In 11 patients $(7.5 \%)$ pain paroxysms remained unchanged postoperatively (detail for patients with multiple sclerosis: nine of 10 immediately pain free; one of 10 unchanged).

Seven patients were lost and long term follow up was available in 141 of 148 cases. Postoperative relief from TN was complete without medication in 105 of 141 cases (75\%); good, with rare and slight paroxysms not requiring medication, in one $(0.7 \%)$; partial, requiring lower dosage of drugs, in 13 (9\%); and absent in 22 $(15.3 \%)$. In detail for patients with multiple sclerosis (follow up 12-39 months, average 24) it was complete in five of 10 , partial in one of 10 , and absent in four of 10 .

Seven patients affected by "essential" TN needed a second MVD during follow up, for a drug resistent recurrence. Complete relief from pain, without medication, was achieved in four cases (findings at repeated surgery: incomplete decompression in two cases; new trigeminal venous compression in one case with sigmoid sinus thrombosis evident at pre-operative MRI, and thick arachnoiditis with a vein adherent to the trigeminal REZ in the remaining case). One patient had a drug resistent recurrence two months after the second MVD (arachnoiditis and venous compression). In two patients pain paroxysm remained unchanged (venous compression in one case, arachnoiditis and venous compression in the other). The general recurrence free curve is shown in figure 1 . To verify the hypothesis that the duration of clinical history was related to the outcome we searched through 1 year, 2 years, 3 years, 4 years, etc. We reached significance at 7 years. Comparison of 


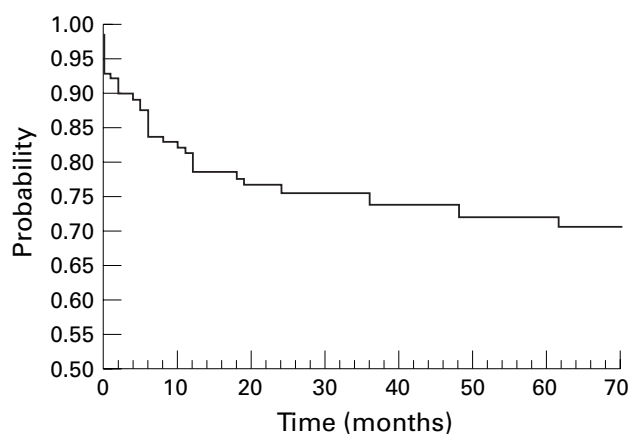

Figure 1 Cumulative proportion of completely pain free patients.

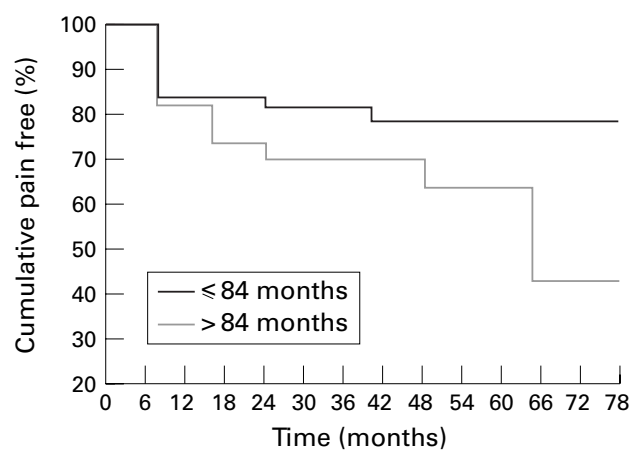

Figure 2 Cumulative probability of remaining pain free by duration of disease before surgery.

cumulative probability of being recurrence free by a shorter or longer than 7 year clinical history is shown in figure 2. No other statistically significant factors predicting long term relief from tic after MDV were identified. A comparison between multiple sclerosis group and "essential" TN group or between cases with a slight compression and a severe compression was not significant because both the multiple sclerosis and slight compression groups were too small (detail for 15 patients with a compression without root distortion: complete relief in 12 of 15 , partial relief in two of 15 , no relief in one of 15$)$.

Operative complications are shown in table 1. There was no surgical mortality in this series. In two patients with a pre-existent severe trigeminal sensorial deficit and atypical neuralgia the disappearance of pain paroxysms was followed by a painful dysaesthesia requiring treatment with tricyclic antidepressant drugs or related medication for burning or aching pain. Out of 87 patients without previous ipsilateral procedures there was a slight permanent trigeminal sensorial deficit in three.

Seventeen patients out of 250 had ipsilateral hearing loss; mild (shown only by postoperative audiometric examination) in nine, and severe (clinically evident) in eight. No patient had permanent facial weakness. Six patients had postoperative diplopia, transient in all cases. A CSF rhinorrhoea required a 3 day external spinal drainage in seven cases and reoperation in five. As shown in table 1, most of the morbidities appeared during the learning period.

Comparison between the incidence of surgical complications in different ages (more than 65 year old $v$ less than 65 year old) did not show any statistically significant difference.

\section{Discussion}

New drugs have been recently introduced in the treatment of trigeminal neuralgia ${ }^{6-10}$ and perhaps the number of surgical candidates will decrease, but carbamazepine and phenytoin

Table 1 Number of operative complications (\%) in 250 consecutive patients who underwent MVD for trigeminal neuralgia ("learning period": cases 1-50). In some cases more than one complication occurred in the same patient

\begin{tabular}{llllc}
\hline & $\begin{array}{l}\text { Cases 1-50 } \\
\text { Year 1990 }\end{array}$ & $\begin{array}{l}\text { Cases 51-196 } \\
\text { Years 1991-Oct 96 }\end{array}$ & $\begin{array}{l}\text { Cases 197-250 } \\
\text { Years Nov 1996-98 }\end{array}$ & $\begin{array}{c}\text { Total } \\
\text { (250 cases) }\end{array}$ \\
\hline Mild hearing loss & $/$ & $6(4)$ & $3(5.5)$ & $9(3.6)$ \\
Severe hearing loss & $5(10)$ & $2(1.3)$ & $1(1.8)$ & $8(3.2)$ \\
Transient diplopia & $3(6)$ & $1(0.7)$ & $2(3.6)$ & $6(2.4)$ \\
Mild transient facial weakness & $2(4)$ & $1(0.7)$ & $/$ & $3(1.2)$ \\
Transient dysphagia & $/$ & $1(0.7)$ & $1(1.8)$ & $1(0.4)$ \\
Cerebellar haematoma & $9(18)$ & $2(1.3)$ & $1(1.8)$ & $1(0.4)$ \\
Cerobrospinal fluid leak & $9(18)$ & $3(2)$ & $1(1.8)$ & $13(5)$ \\
Mild transient facial numbness & $1(2)$ & $1(0.7)$ & $1(1.8 \%)$ & $3(1.2)$ \\
Mild permanent facial numbness & $/$ & $/$ & & $1(0.4 \%)$ \\
Sigmoid sinus thrombosis & & & & \\
\hline
\end{tabular}

^Revealed only by postoperative audiometric examination

Table 2 Completely pain free patients after MVD for trigeminal neuralgia

\begin{tabular}{|c|c|c|c|c|}
\hline Author(s) & No of patients & $\mathrm{No}(\%)$ of CPFPs & Significant recurrence (\%) & Follow up (mean) \\
\hline Taarnhoj $^{13}(1982)$ & 350 & $225(64.3)$ & $113(32.3)$ & Up to11.5 y \\
\hline Szapiro et $a l^{14}(1985)$ & 68 & $56(82)$ & $2(3)$ & $1-5 y$ \\
\hline Burchiel et al ${ }^{15}(1988)$ & 36 & $19(53)$ & $11(30)$ & $7.5-11.5$ y $(8.5$ y) \\
\hline Bederson and Wilson ${ }^{16}(1989)$ & 252 & $189(75)$ & $44(17)$ & $0.5-16$ y $(5 \mathrm{y})$ \\
\hline Dahle et $\mathrm{l}^{17}$ (1989) & 54 & $43(79)$ & $11(21)$ & $3-7$ y (3.1 y) \\
\hline Sindou et $\mathrm{l}^{18}$ (1990) & 60 & $50(83)$ & $2(3)$ & /-(16 months $)$ \\
\hline Klun $^{19}(1992)$ & 178 & $167(94)$ & $5(3)$ & $0.5-12$ y $(5.2 \mathrm{y})$ \\
\hline Yamaki et alo $(1992)$ & 60 & $38(63)$ & $9(15)$ & $0.5-5.5 \mathrm{y}$ \\
\hline Sindou et al $l^{11}(1993)$ & 420 & /-(91) & - $-(6)$ & ? \\
\hline Sun $e t a l^{22}(1994)$ & 61 & $46(75)$ & $10(16)$ & $1-10$ y (80 months) \\
\hline Mendoza and Illingworth ${ }^{23}$ (1995) & 133 & $95(71)$ & $18(13)$ & $0.5-15$ y $(5.3$ y) \\
\hline Barker et $a l^{24}(1996)$ & 1185 & $903(76)$ & $282(24)$ & $1-20 \mathrm{y}(6.2 \mathrm{y})$ \\
\hline Kondo $^{25}(1997)$ & 281 & $244(87)$ & $23(8)$ & $>5 y$ \\
\hline Liao et $a l^{26}(1997)$ & 80 & ? & 5 & $0.75-4 \mathrm{y}$ \\
\hline Coakham and $\operatorname{Moss}^{27}$ (1998) & $>150$ & ? & $/-(10)$ & Up to $17 \mathrm{y}$ \\
\hline Present report (1998) & 141 & $105(74)$ & $22(15.6)$ & $1-6$ y $(3 y)$ \\
\hline
\end{tabular}

$\mathrm{CPFPs}=$ completely pain free patients. 
Table 3 MVD: mortality and long term side effects

\begin{tabular}{|c|c|c|c|c|c|c|c|c|}
\hline Author(s) & $\begin{array}{l}\text { No of } \\
\text { patients }\end{array}$ & Mortality & Cereb Inf & Def $V I I I^{\circ}$ & Def $V I I^{\circ}$ & Dipl & $\operatorname{Def} V^{\circ}$ & $P D$ \\
\hline Taarnhoj $1^{3}(1982)$ & 350 & $2(1.1 \%)$ & $0.3 \%$ & $1.4 \%$ & $0.6 \%$ & $0.3 \%$ & 0 & 0 \\
\hline Barba and Alksne ${ }^{29}(1984)$ & 37 & 0 & 0 & 0 & 0 & 0 & $5 \%$ & 0 \\
\hline Zorman and Wilson ${ }^{31}(1984)$ & 125 & 0 & 0 & $3 \%$ & 0 & 0 & 0 & 0 \\
\hline Szapiro et $\mathrm{al}^{14}(1985)$ & 70 & $1(1.43 \%)$ & $1.4 \%$ & 0 & 0 & 0 & 0 & 0 \\
\hline Bederson and Wilson ${ }^{16}(1989)$ & 252 & $2(0.07 \%)$ & 0 & $3 \%$ & 0 & 0 & 0 & 0 \\
\hline Dahle et $a l^{17}(1989)$ & 57 & $1(1.7 \%)$ & 0 & 0 & 0 & 0 & $1.7 \%$ & $1.7 \%$ \\
\hline Sindou et $a l^{18}(1990)$ & 60 & 0 & 0 & 0 & 0 & 0 & 0 & 0 \\
\hline 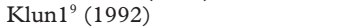 & 220 & $3(1.3 \%)$ & 0 & $0.4 \%$ & 0 & 0 & 0 & 0 \\
\hline Sun et $a l^{22}(1994)$ & 61 & 0 & 0 & $1.5 \%$ & 0 & 0 & $1.5 \%$ & $1.5 \%$ \\
\hline Meneses et $a l^{22}$ (1995) & 50 & 0 & 0 & 0 & 0 & 0 & 0 & \\
\hline Pamir et $a l^{33}$ (1995) & 32 & 0 & $3 \%$ & 0 & 0 & 0 & 0 & 0 \\
\hline Mendoza and Illingworth ${ }^{23}$ (1995) & 133 & $1(0.7 \%)$ & $1.4 \%$ & 0 & 0 & 0 & 0 & 0 \\
\hline Barker et $\mathrm{l}^{4}(1996)$ & 1336 & $2(0.2 \%)$ & $0.1 \%$ & $1 \%$ & 0 & 0 & 0 & 0 \\
\hline Present report (1998) & 250 & 0 & $0.4 \%$ & $3.2 \%$ & 0 & 0 & $1.2 \%$ & 0 \\
\hline
\end{tabular}

Cereb inf=cerebellar infarct; Def=deficit; Dipl=diplopia; $P D=$ painful dysaesthesia.

are the only drugs with established efficacy. Due to relapse about half of all patients eventually require an operation for pain relief. ${ }^{11}$ No other selection criteria for surgery than refractoriness to medical therapy was used in case of typical trigeminal "tic douloureux". MVD was proposed to patients affected by MS only in the absence of plaques demonstrated by MRI in the trigeminal REZ, pontine tract, and nuclei. MRI can be an extremely accurate tool for detecting neurovascular compression, ${ }^{12}$ but the absence of an MRI identified neurovascular compression should not exclude the patient with intractable pain from open surgery because of the possibility of false negative MRI studies, even with high definition MR tomographic angiography (personal observation and Meany $e t a l^{12}$ ).

In the literature there are several series reporting data on the efficacy of MVD (table 2). Direct comparison of results is difficult because of different durations of follow up and differing definitions of operative success. Considering the number of completely pain free patients at long term follow up, our results ( $74 \%$ of completely pain free patients at 5 year follow up) are in the range (53\%-94\%) of other reported series and in particular are similar to those of Barker and Jannetta ${ }^{24}$ and of other series using actuarial methods. ${ }^{15} 1623$ Similar results (39 of 50 patients completely pain free at 7 year follow up), but with a higher rate of early re-exploration for inadequate decompression (four patients successfully reoperated on within the first month for persistent pain), were achieved in the first 50 cases, although with a slightly different surgical technique. Coakham and Moss $^{27}$ recently reported better results, with only a $10 \%$ relapse rate in their 17 year follow up study of more than 150 patients. They achieved such results using the Teflon sling technique developed by Fukushima. ${ }^{28}$

Female sex, ${ }^{416}$ operative findings at microvascular decompression, ${ }^{15}{ }^{19}$ and previous destructive surgical treatments ${ }^{16}{ }^{29}$ have been correlated with outcome by others: in this series we did not find them to be prognostic variables. In our study a history of more than 7 years was the only statistically significant prognostic factor $(p<0.05)$, suggesting that root damage and abnormal neural hyperactivity might become irreversible as it has been shown in experimen- tal kindling. ${ }^{30}$ Arterial hypertension, which theoretically could enhance the possibility of a new vascular contact, did not affect the results.

Some of the criticisms about MVD are based on presumed high mortality and morbidity. In our series there was no mortality and no permanent morbidity. Ataxia, disequilibrium, and gait disturbances sometimes found in the early postoperative period, at hospital discharge (3 days after operation), fully recovered within 2 weeks without rehabilitation. Collecting data from the literature series on more than 3000 published cases (table 3) the mortality rate is $0.3 \%$ (12 of 3033). Cranial nerve morbidity is reported, but generally diplopia, dysphagia, facial weakness, vertigo, and trigeminal hypoaesthesia are all transient. Injury to the acoustic branch of the VIII cranial nerve is the only relevant long term cranial nerve dysfunction reported in several series, ranging from $0.1 \%$ to $3 \%$ (table 3 ). We recorded brain stem acoustic evoked potentials during MVD in 98 consecutive procedures. Although no clear correlation could be found between the increase in latency, the reduction in amplitude and the degree of hearing loss, ${ }^{5}$ a direct relation between some surgical manoeuvres and brain stem acoustic evoked potentials significant changes was found and led us to a mini-invasive supracerebellar approach. In the 200 cases operated on through this approach, we had only three cases of severe hypoacusia, always related to a particular surgical problem (bleeding from petrous bridge vein in one case, troublesome dissection from the pons of a dolicomegabasilar artery invaginating the trigeminal and the facial nerve in two cases). Probably this is the only complication that cannot be prevented in all cases because of the extreme vulnerability of the internal auditory artery and its coclear branches. Other reported complications such as CSF leakage, haemotympanum, sigmoid sinus thrombosis, cerebellar infarct, and haematoma can be avoided with a careful surgical technique and perfect haemostasis. In a patient in our series, sigmoid sinus thrombosis was found associated with a new venous trigeminal compression. We did not find any age related statistically significant difference in incidence of surgical complications and so we perform MVD without an absolute age limit. Furthermore, in elderly patients surgical exposure of cerebellopontine 
angle is easier due to atrophy, and the postoperative course was generally uneventful with early mobilisation.

Among other less invasive surgical procedures, but all causing more or less controlled injury to the trigeminal nerve, root, or ganglion, only radiofrequency rhizotomy, as recently pointed out by Taha and Tew, ${ }^{34}$ has pain recurrence rates similar to that of MVD. Unfortunately this result can be accomplished only at the cost of a sensorial deficit which is far from negligible and not well tolerated in some patients.

Multiple sclerosis has long been considered a contraindication to MVD because of supposed origin from a demyelinating plaque in the central trigeminal pathways ${ }^{35}$ or in the trigeminal REZ. ${ }^{36}$ In the literature only a few patients with multiple sclerosis who underwent MVD for trigeminal neuralgia can be found and results are contradictory. ${ }^{37}{ }^{38}$ In our series five of 10 patients did well with a follow up of 12-39 months. Although the small series and the short follow up may not allow definitive conclusions, our results suggest that it may be worthwhile not depriving patients with MS of a potential cure. Further studies are needed to better evaluate the role of MVD for trigeminal neuralgia in patients with MS.

A peripheral hypothesis, ${ }^{39} 40$ central hypothesis, ${ }^{41}$ and more recently, theories trying to reconcile central and peripheral hypotheses ${ }^{42} 43$ about aetiopathogenesis of trigeminal neuralgia have been invoked. Nevertheless it remains a mystery. Vascular cross compression is now increasingly accepted as an important aetiological factor. We found a vascular contact in all cases, even in patients with multiple sclerosis. Sometimes the involved vessels are subtle and the root does not seem grossly compressed. In reoperated cases a missed vessel, a new compressing vessel (an intratrigeminal vein in a patient in our series with sigmoid sinus trombosis at preoperative MRI) or a teflon mediated compression can be seen, but sometimes only an arachnoiditis is found. A similar arachnoiditis can be found when reoperating on patients, previously operated on for lesions in the cerebellopontine angle and who do not have trigeminal neuralgia. This apparent contradiction and many others (different rates of vascular compressions in different series, patients having trigeminal neuralgia with little or no vascular compression, evidence of vascular compression without neuralgia, vascular compression of the motor branch without hemimasticatory spasm, patients certainly well decompressed with persistent pain etc ${ }^{4}{ }^{15}$ ) can be partially explained by different patient selection and different observers. Collectively they mean that the problem of neurovascular compression cannot be considered as a simple cause-effect mechanism. A central involvement of trigeminal pathways with a delicate balance between abnormal and physiological imputs coming from the damaged root and modified excitability of neurons of the trigeminal nucleus must also be considered. The concept of a central neuromodulatory role of impulses coming from the area of cross compression also explains the possibility that a long lasting alteration of discharge modalities of the trigeminal root can cause lowering of the pain threshold as suggested by recent reports on extracranial neurovascular conflicts. ${ }^{44}{ }^{45}$ MVD certainly acts on pain modulation by peripheral pathological impulses and even if it could not be considered as the "definitive aetiological cure" 34 it certainly is one of the best rational therapheutic options for treatment of trigeminal neuralgia.

\section{Conclusions}

According to our large experience with both open surgery and percutaneous techniques, ${ }^{46}$ MVD is a safe and effective method for relieving typical TN in patients of all ages. It should be proposed as first choice surgery to all patients affected by TN. Preoperative MRI examination is mandatory to exclude nonvascular trigeminal compression. Sigmoid sinus thrombosis can affect outcome by causing a new venous compression. The recurrence rate seems to be higher in patients with MS and in those patients with a longer clinical history.

We thank Dr A Solari and Miss M Farinotti for statistical analysis. We are grateful to Miss Lucy Wachendorfer for her assistance in reviewing the English manuscript.

1 Dandy WE. Concerning the cause of trigeminal neuralgia. Am ₹ Surg 1934;24:447-55.

2 Gardner WJ, Miklos MV. Response of trigeminal neuralgia to decompression of sensory root. Discussion of cause of to decompression of sensory root. Discussion

3 Jannetta PJ. Arterial compression of the trigeminal nerve at Jannetta PJ. Arterial compression of the trigeminal nerve at
the pons in patients with trigeminal neuralgia. $\mathcal{F}$ Neurosurg 1967;26:159-62.

4 Adams CBT. Microvascular decompression: an alternative view and hypothesis. F Neurosurg 1989;57:1-12.

5 Broggi G, Scaioli V, Brock S, et al. Neurophysiological monitoring of cranial nerves during posterior fossa surgery. Acta Neurochir Suppl (Wien) 1995;64:35-9.

6 Farago F. Trigeminal neuralgia: its treatment with two new carbamazepine analogues. Eur Neurol 1987;26:73-83.

7 Fromm GH, Terrence CF. Comparison of L-baclofen and racemic baclofen in trigeminal neuralgia. Neurology 1987; 37:1725-8.

8 Lechin F, Van Der Dijs B, Amat J. Definite and sustained improvement with pimozide of two patients with severe trigeminal neuralgia. $\mathcal{F}$ Med 1988;19:243-56.

9 Lindstrom P, Lindblom U. The analgesic effect of tocainide in trigeminal neuralgia. Pain 1987;28:45-50.

10 Vilming ST, Lyberg T, Lastate X. Tizanidine in the management of trigeminal neuralgia. Cephalalgia 1986;6: $181-2$.

11 Katusic S, Beard CM, Bergstralh E, et al. Incidence and clinical features of trigeminal neuralgia, Rochester, Minnesota, 1945-84. Ann Neurol 1990;27:89-95.

12 Meaney JFM, Eldridge PR, Dunn LT, et al. Demonstration of neurovascular compression in trigeminal neuralgia with magnetic resonance imaging. I Neurosurg 1995;83:799805 .

13 Taarhoj P. Decompression of the posterior trigeminal root in trigeminal neuralgia: a 30-year follow-up review. 7 Neurosurg 1982;57:14-17.

14 Szapiro J, Sindou M, Szapiro J. Prognostic factors in microvascular decompression for trigeminal neuralgia. Neurosurg 1985;17:920-9.

15 Burchiel KJ, Clarke H, Haglund M, et al. Long term efficacy of microvascular decompression in trigeminal neuralgia. $\mathfrak{F}$ Neurosurg 1988;69:35-8

16 Bederson JB, Wilson CB. Evaluation of microvascular decompression and partial sensory rhizotomy in 252 cases of trigeminal neuralgia. I Neurosurg 1989;71:359-67.

17 Dahle L, Von Essen C, Kourtopoulos H, et al. Microvascular decompression for trigeminal neuralgia. Acta Neurochir (Wien) 1989;99:109-12.

18 Sindou M, Amrani F, Mertens P. Decompression vasculaire microchirurgicale pour nèvralgie du trijumeau. Comparaison de deux modalitès techniques et dèduction physiopathologiques. Etude sur 120 cas. Neurochirurgie 1990;36: $16-26$.

19 Klun B. Microvascular decompression and partial sensory rhizotomy in the treatment of trigeminal neuralgia: personal experience with 220 patients. Neurosurg 1992;30: 49-52.

20 Yamaki T, Hashi K, Niwa J, et al. Results of reoperation for failed microvascular decompression. Acta Neurochir (Wien) 1992;115:1-7. 
21 Sindou M, Mertens P. Microsurgical vascular decompression in trigeminal and glosso-vago- pharyngeal neuralgias. A 20 year exp

22 Sun T, Saito S, Nakai O, et al. Long-term results of microvascular decompression for trigeminal neuralgia with reference to probability of recurrence. Acta Neurochir (Wien) 1994;126:144-8

23 Mendoza N, Illingworth RD. Trigeminal neuralgia treated by microvascular decompression: a long term follow-up study. Br $\mathcal{F}$ Neurosurg 1995;9:13-19.

24 Barker FG, Jannetta JJ, Bissonette DJ, et al. The long term outcome of microvascular decompression for trigeminal neuralgia. N Engl f Med 1996;334:1077-83.

25 Kondo A. Follow-up results of microvascular decompression in trigeminal neuralgia and hemifacial spasm. Neurosurgery 1997;40:46-52.

26 Liao JJ, Cheng WC, Chang CN, et al. Reoperation for recurrent trigeminal neuralgia after microvascular decompression. Surg Neurol 1997;47:562-70.

pression. Surg Neurol 1997;47:562-70. [letter]. F Neurosurg 1998;88:617-18.

28 Fukushima T. Operative treatment of hemifacial spasm. Clinical Neuroscience ( $(p n)$ 1986;4:112-13.

29 Barba D, Alksne JF. Success of microvascular decompression with and without prior surgical therapy for trigeminal neuralgia. F Neurosurg 1984;60:104-7.

30 Goddard GV, McIntyre DC, Leech CK. A permanen change in brain function resulting from daily electrical stimulation. Exp Neurol 1969;25:295-330.

31 Zorman G, Wilson CB. Outcome following microvascular decompression or partial sensory rhizotomy in 125 cases of trigeminal neuralgia. Neurology 1984;34:1362-5.

32 Meneses MS, Clemente R, Russ HHA, et al. Traitment microchirurgical de la nevvralgie du trijumeau: étude sur 50 cases. Neurochirurgie 1995;41:349-52.

33 Pamir MN, Zirh TA, Ozer AF, et al. Microvascular decompression in the surgical management of trigeminal neuralgia. Neurosurg Rev 1995;18:163-7.
34 Taha JM, Tew JM. Comparison of surgical treatments for trigeminal neuralgia: reevaluation of radiofrequency rhizotrigeminal neuralgia: reevaluation of

35 Loeser JD. Trigeminal neuralgia and multiple sclerosis [comment]. Neurosurgery 1979;5:716.

36 Lazar ML, Kirkpatrick JB. Trigeminal neuralgia and multiple sclerosis: demonstration of the plaque in an operative case. Neurosurgery 1979;5:711-15.

37 Resnick DK, Jannetta PJ, Lunsford LD, et al. Microvascular decompression for trigeminal neuralgia in patients with multiple sclerosis. Surg Neurol 1996;46:358-62.

38 Meaney JFM, Watt JWG, Eldridge PR, et al. Association between trigeminal neuralgia and multiple sclerosis: role of magnetic resonance imaging. 7 Neurol Neurosurg Psychiatry 1995;59:253-9.

39 Kerr FWL. Evidence for a pheripheral etiology of trigeminal neuralgia. $\mathcal{f}$ Neurosurg $1967 ; 26: 168-74$.

40 Rappaport ZH, Devor M. Trigeminal neuralgia: the role of sappaport $\mathrm{ZH}$, Devor $\mathrm{M}$. Trigeming neuralgia: the role of 1994;56:127-38.

41 Dubner R, Sharav Y, Gracely RH, et al. Idiopathic trigeminal neuralgia: sensory features and pain mechanisms. Pain

42 Fromm GH, Terrence CF, Maroon JC. Trigeminal neuralgia: current concepts regarding etiology and pathogenesis. Arch Neurol 1984;41:1204-7.

43 Pagni CA. The origin of tic douloureux: a unified view. $\mathcal{F}$ Neurosurg Sci 1993;37:185-94.

44 Franzini A, Scaioli V, Leocata F, et al. Pain syndrome and focal myokymia due to anterior interosseous neurovascular relationships: report of a case and neurophysiological considerations. F Neurosurg 1995;82:578-80.

45 Scaioli V, Franzini A, Leocata F, et al. Hand dystonia and neuralgic pain due to neurovascular contact to cervical spinal root [letter]. Mov Disord 1996;11:102-4.

46 Broggi G, Franzini A, Lasio G, et al. Long-term results of percutaneous retrogasserian thermorhizotomy for essential percutaneous retrogasserian thermorhizotomy for essential patients. Neurosurgery 1990;26:783-7. 